On quadrature methods for highly oscillatory integrals and their implementations

by

Arieh Iserles and Syvert P. Nørsett

\author{
PREPRINT \\ NUMERICS NO. 6/2004 \\ ISSN: 0804-9181
}

NORWEGIAN UNIVERSITY OF SCIENCE AND

TECHNOLOGY

TRONDHEIM, NORWAY 


\title{
ON QUADRATURE METHODS FOR HIGHLY OSCILLATORY INTEGRALS AND THEIR IMPLEMENTATION *
}

\author{
A. ISERLES ${ }^{1}$ and S.P. NØRSETT ${ }^{2}$ \\ ${ }^{1}$ Department of Applied Mathematics and Theoretical Physics, Centre for Mathematical \\ Sciences, University of Cambridge, Wilberforce Rd, Cambridge CB3 0WA, United Kingdom. \\ email: ai@damtp.cam.ac.uk \\ ${ }^{2}$ Department of Mathematical Sciences, Norwegian University of Science and Technology \\ N-7491 Trondheim, Norway. \\ email: norsett@math.ntnu.no
}

\begin{abstract}
.
The main theme of this paper is the construction of efficient, reliable and affordable error bounds for two families of quadrature methods for highly oscillatory integrals. We demonstrate, using asymptotic expansions, that the error can be bounded very precisely indeed at the cost of few extra derivative evaluations. Moreover, in place of derivatives it is possible to use finite difference approximations, with spacing inversely proportional to frequency. This renders the computation of error bounds even cheaper and, more importantly, leads to a new family of quadrature methods for highly oscillatory integrals that can attain arbitrarily high asymptotic order without computation of derivatives.
\end{abstract}

\section{Asymptotic and Filon-type methods}

The theme of this paper is the efficient quadrature of highly oscillatory integrals of the form

$$
I[f]=\int_{a}^{b} f(x) \mathrm{e}^{\mathrm{i} \omega g(x)} \mathrm{d} x,
$$

where $-\infty<a<b<\infty$, both $f$ and $g$ are sufficiently smooth functions and $|\omega|$ is large. Such integrals feature in a very wide range of applications, underscoring the importance of efficient and reliable means of their computation. Yet, a pervasive point of view among both numerical analysts and workers in application areas is that the computation of (1.1) is exceedingly difficult and that the cost steeply increases with $|\omega|$.

In [5] we set out to challenge this point of view. Although Gauss-Christoffel quadrature, the classical means to compute integrals, completely fails when $1 /|\omega|$ is significantly smaller than the number of quadrature points, this represents a shortcoming of the method, rather than an intrinsic 'difficulty' in computing $I[f]$. In place of Gauss-Christoffel, we have advocated two new, altogether different methods: the asymptotic method and the Filon-type method. Both methods match leading terms in the asymptotic expansion of $I[f]$ in inverse powers of $\omega$. Therefore - and this might come as a surprise to all those conditioned by

*Received ???. Communicated by ???. 
the urban legend that highly oscillatory problems are difficult to compute the quality of these methods increases in tandem with oscillation. Indeed, we can make this improvement in precision arbitrarily large by using derivative information at designated points.

The purpose of the current paper is threefold. Firstly, we present a method of error control of highly oscillatory quadrature which is both affordable and exceedingly precise. Secondly, we use our error estimates to clarify why, as observed in [5], in an overwhelming majority of cases Filon-type quadrature is substantially more precise than asymptotic quadrature. Finally, guided by the logic of our error estimates, we present a new method for the quadrature of highly oscillatory integrals which, while producing an error commensurate in quality with asymptotic and Filon-type methods, does not require the computation of derivatives.

We commence by reviewing briefly relevant material from [5]. As will be familiar to experts in asymptotic analysis, an important distinction which we need to make at the outset is between oscillators $g$ such that $g^{\prime} \neq 0$ in $[a, b]$ and oscillators with stationary points $d_{1}, d_{2}, \ldots, d_{s} \in(0,1), s \geq 1$, where the derivative vanishes $[6,8]$.

If $g^{\prime} \neq 0$ in $[a, b]$ then it follows from the van der Corput lemma that $I[f]=$ $O\left(\omega^{-1}\right),|\omega| \rightarrow \infty[8]$. More specifically, an explicit asymptotic expansion of $I[f]$ can be derived relatively easily by repeated integration by parts. Thus, we let

$$
\begin{aligned}
f_{0}(x) & =f(x), \\
f_{m+1}(x) & =\frac{\mathrm{d}}{\mathrm{d} x} \frac{f_{m}(x)}{g^{\prime}(x)}, \quad m \in \mathbb{Z}_{+},
\end{aligned}
$$

whence

$$
I[f] \sim-\sum_{m=0}^{\infty} \frac{1}{(-\mathrm{i} \omega)^{m+1}}\left[\frac{\mathrm{e}^{\mathrm{i} \omega g(b)}}{g^{\prime}(b)} f_{m}(b)-\frac{\mathrm{e}^{\mathrm{i} \omega g(a)}}{g^{\prime}(a)} f_{m}(a)\right], \quad|\omega| \gg 1 .
$$

Given $p \in \mathbb{N}$, we thus define the asymptotic method

$$
Q_{p}^{\mathrm{A}}[f]=-\sum_{m=0}^{p-1} \frac{1}{(-\mathrm{i} \omega)^{m+1}}\left[\frac{\mathrm{e}^{\mathrm{i} \omega g(b)}}{g^{\prime}(b)} f_{m}(b)-\frac{\mathrm{e}^{\mathrm{i} \omega g(a)}}{g^{\prime}(a)} f_{m}(a)\right] .
$$

We note at once that

$$
Q^{\mathrm{A}}[f]-I[f] \sim O\left(\omega^{-p-1}\right), \quad|\omega| \gg 1,
$$

therefore (1.3) is of asymptotic order $p$. Since each $f_{m}$ is a linear combination of $f, f^{\prime}, \ldots, f^{(m)}$, the formation of $Q^{\mathrm{A}}[f]$ requires the computation of $f^{(k)}(a)$ and $f^{(k)}(b), k=0,1, \ldots, p-1$. This underscores our assertion that good methods for highly oscillatory quadrature improve their performance once frequency $|\omega|$ becomes larger, as well as providing a clear-cut explanation of this phenomenon: unlike Gauss-Christoffel methods which, in common with most discretization methods, are based on reproduction of polynomials, i.e. on the ansatz that the solution is well approximated by a Taylor expansion, the assumption underlying (1.3) is that $I[f]$ is approximated well by the asymptotic expansion (1.2). 
An alternative to (1.3) is to use a Filon-type method, which considerably extends an idea originally due to Louis Napoleon Georges Filon [3]. Let

$$
a=c_{1}<c_{2}<\cdots<c_{\nu-1}<c_{\nu}=b
$$

be $\nu \geq 2$ prescribed nodes in the interval $[a, b]$. Having chosen multiplicities $m_{1}, m_{2}, \ldots, m_{\nu} \in \mathbb{N}$, we let $\tilde{f}$ be the $m$ th degree polynomial, where $m=$ $\sum_{k=1}^{\nu} m_{k}-1$, such that

$$
\tilde{f}^{(l)}\left(c_{k}\right)=f^{(l)}\left(c_{k}\right), \quad l=0,1, \ldots, m_{k}-1, \quad k=1,2, \ldots, \nu .
$$

Note that the Hermite interpolation polynomial $\tilde{f}$ is unique. Assuming that the moments

$$
\mu_{k}(\omega)=\int_{a}^{b} x^{k} \mathrm{e}^{\mathrm{i} \omega g(x)} \mathrm{d} x, \quad k=0,1, \ldots, m,
$$

can be calculated explicitly, we let $p=\min \left\{m_{1}, m_{\nu}\right\}$ and define

$$
Q_{p}^{\mathrm{F}}[f]=\int_{a}^{b} \tilde{f}(x) \mathrm{e}^{\mathrm{i} \omega g(x)} \mathrm{d} x .
$$

The above Filon-type method, like (1.3), is of asymptotic order $p$. The proof is exceedingly easy and is fundamental to the work of the present paper. Thus, using (1.2) on $h=\tilde{f}-f$,

$$
\begin{aligned}
Q_{p}^{\mathrm{F}}[f]-I[f] & =I[\tilde{f}]-I[f]=I[h] \\
& \sim \sum_{m=0}^{\infty} \frac{1}{(-\mathrm{i} \omega)^{m+1}}\left[\frac{\mathrm{e}^{\mathrm{i} \omega g(b)}}{g^{\prime}(b)} h_{m}(b)-\frac{\mathrm{e}^{\mathrm{i} \omega g(a)}}{g^{\prime}(a)} h_{m}(a)\right], \quad|\omega| \gg 1
\end{aligned}
$$

However, since each $h_{m}$ is a linear combination of $h, h^{\prime}, \ldots, h^{(m)}$, it follows from interpolation conditions at $a$ and $b$ that $h^{(k)}(a)=h^{(k)}(b)=0, k=0,1, \ldots, p-1$. This proves that $Q^{\mathrm{F}}[f]-I[f]=O\left(\omega^{-p-1}\right)$, hence asymptotic order $p$.

The situation is different once $g$ is allowed stationary points in $(a, b)$. We may assume without loss of generality that $g^{\prime}$ vanishes at a single point in $(a, b)$ : given any finite number of stationary points, we may divide $(a, b)$ into subintervals with a single stationary point in each. Thus, we assume that

$$
g^{\prime}(d)=g^{\prime \prime}(d)=\cdots=g^{(s-1)}(d)=0, \quad g^{(s)}(d) \neq 0,
$$

where $d \in(a, b)$ and $s \geq 2$. It is known from the classical method of stationary phase that for every smooth function $f$ with compact support in the neighbourhood of $d$ there exists an asymptotic expansion

$$
I[f] \sim \omega^{1 / s} \sum_{m=0}^{\infty} A_{m}[f](\omega) \omega^{-m / s}, \quad|\omega| \gg 1,
$$

where each functional $A_{m}[f]$ is a periodic function of $\omega$. The first functional, $A_{0}[f]$, is known explicitly but, unfortunately, explicit expressions are not available for all $A_{m}[f], m \geq 1$. This renders a truncation of (1.6), following the 
logic of the asymptotic method (1.3), unusable from the numerical standpoint. (Another obstacle, the need for $f$ to have sufficiently small support near $d$, can be avoided by using a technique described in [4].)

An alternative to the method of stationary phase has been introduced in [5]. For simplicity we present it just for the case $s=2$, noting that it easily scales up to all $s \geq 2$, albeit at the cost of more complicated expressions. Thus, let

$$
\begin{aligned}
f_{0}(x) & =f(x) \\
f_{m+1}(x) & =\frac{\mathrm{d}}{\mathrm{d} x} \frac{f_{m}(x)-f_{m}(d)}{g^{\prime}(x)}, \quad m \in \mathbb{Z}_{+} .
\end{aligned}
$$

Note that the singularity on the right is removable, thus each $f_{m}$ is smooth, provided that $f$ is so. Moreover, in $(a, b) \backslash\{d\}$ each $f_{m}$ is a linear combination of $f^{(k)}, k=0,1, \ldots, m$, while $f_{m}(d)$ is a linear combination of $f^{(k)}(d), k=$ $0,1, \ldots, 2 m$.

It is possible to prove that

$$
\begin{array}{ll}
I[f] \sim & -\sum_{m=0}^{\infty} \frac{1}{(-\mathrm{i} \omega)^{m+1}}\left\{\frac{\mathrm{e}^{\mathrm{i} \omega g(b)}}{g^{\prime}(b)}\left[f_{m}(b)-f_{m}(d)\right]-\frac{\mathrm{e}^{\mathrm{i} \omega g(a)}}{g^{\prime}(a)}\left[f_{m}(a)-f_{m}(d)\right]\right\} \\
(1.7) \quad+\mu_{0}(\omega) \sum_{m=0}^{\infty} \frac{1}{(-\mathrm{i} \omega)^{m}} f_{m}(d), \quad|\omega| \gg 1 .
\end{array}
$$

We note that (1.7) is a generalization of the asymptotic expansion (1.2) and that its terms can be generated recursively in an explicit form. However, unlike (1.6), this is not an asymptotic expansion in the usual sense, since the function $\mu_{0}$, which we assume to be explicitly known, is retained. This also explains why no fractional powers of $\omega$ feature explicitly in (1.7): they are "hidden" inside $\mu_{0}$.

Once (1.7) is truncated, we obtain the asymptotic method

$$
\begin{aligned}
& Q_{p}^{\mathrm{A}}[f]=-\sum_{m=0}^{p-1} \frac{1}{(-\mathrm{i} \omega)^{m+1}}\left\{\frac{\mathrm{e}^{\mathrm{i} \omega g(b)}}{g^{\prime}(b)}\left[f_{m}(b)-f_{m}(d)\right]-\frac{\mathrm{e}^{\mathrm{i} \omega g(a)}}{g^{\prime}(a)}\left[f_{m}(a)-f_{m}(d)\right]\right\} \\
&(1.8) \quad+\mu_{0}(\omega) \sum_{m=0}^{p-1} \frac{1}{(-\mathrm{i} \omega)^{m}} f_{m}(d)
\end{aligned}
$$

and it follows at once that the asymptotic order is $p-\frac{1}{2}$. (Alternatively, we can sum up to $p$ in the second sum, whence the asymptotic order becomes $p$.) Note that the computation of (1.8) requires $p-1$ derivatives of $f$ at the endpoints and $2 p-2$ derivatives at the stationary point $d$.

A generalization of a Filon-type method is fairly straightforward. Thus, we choose $\nu \geq 3$ distinct nodes in $[a, b]$ and require that both the endpoints and the stationary point are among them. Thus, $c_{1}=a, c_{\nu}=b$ and there exists $r \in\{2,3, \ldots, \nu-1\}$ such that $c_{r}=d$. Again, $m_{k} \geq 1, k=1,2, \ldots, \nu$, and we stipulate that $m_{r} \geq 2 p-1$, where $p=\min \left\{m_{1}, m_{\nu}\right\}$. Let an $m$ th degree polynomial $\tilde{f}$, where $m=\sum_{k=1}^{\nu} m_{k}-1$, satisfy the Hermite interpolation conditions (1.4). The Filon-type method $Q_{p}^{\mathrm{F}}[f]$ is then given by (1.5) and it follows at once from our method of proof that its asymptotic order is also $p-\frac{1}{2}$. 


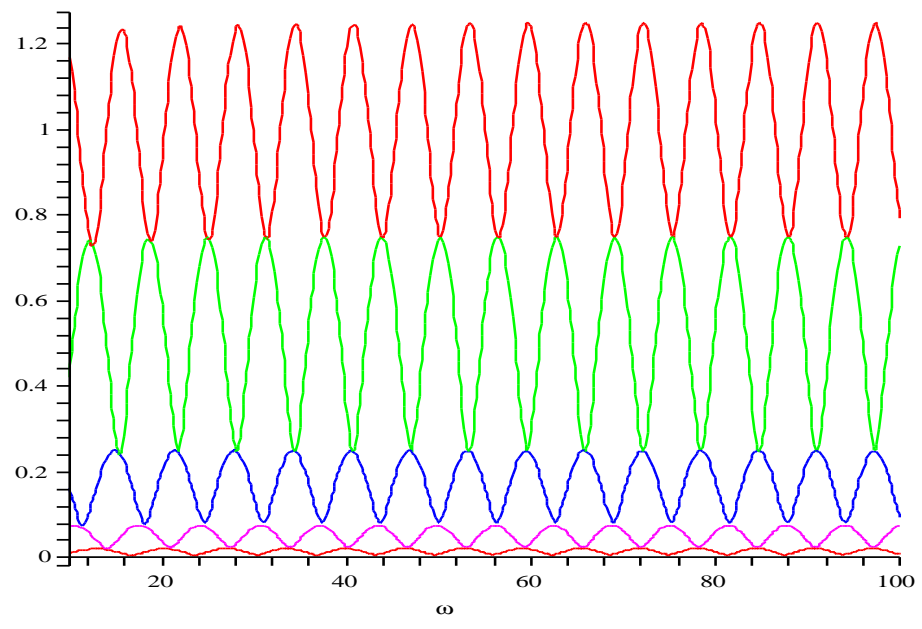

Figure 1.1: The error for $Q_{1}^{\mathrm{A}}$ (top) and four different Filon-type methods $Q_{1}^{\mathrm{F}}$ (from the second plot down), scaled by $\omega^{2}$, for $f(x)=1 /(1+x), g(x)=x$, $[a, b]=[0,1]$ and $10<\omega<100$.

Whether stationary points are present or not, we have a dichotomy between an asymptotic method and a Filon-type method. They require the same information (at least for the "minimalist" variant of $Q_{p}^{\mathrm{F}}$, using data only at the endpoints and stationary points) and deliver the same asymptotic order. Since the asymptotic method has typically simpler coefficients, it is natural to ask why should we bother at all with Filon-type methods. A hint into the answer is provided in Fig. 1.1. We display there the error of five methods, all of which have an asymptotic order 1 , as applied to the computation of

$$
\int_{0}^{1} \frac{\mathrm{e}^{\mathrm{i} \omega x}}{1+x} \mathrm{~d} x
$$

All the errors are in absolute value, scaled by $\omega^{2}$, hence according to our theory they should be asymptotically periodic: this is certainly confirmed. However, it is apparent that the size of the error - or, for that matter, the size of the periodic function $e(\omega)$ such that the error is asymptotically $e(\omega) / \omega^{2}$ - differs for different methods. Starting from the top (i.e., the worst) method, we have $Q_{1}^{\mathrm{A}}$, followed by four Filon-like methods: firstly with $c=[0,1]$, then $c=\left[0, \frac{1}{2}, 1\right]$, $\boldsymbol{c}=\left[0, \frac{1}{3}, \frac{2}{3}, 1\right]$ and, finally, at the bottom, $\boldsymbol{c}=\left[0, \frac{1}{4}, \frac{1}{2}, \frac{3}{4}, 1\right]$. Clearly, the more nodes we add, the better the method: the improvement in the "bottom" method, in comparison with $Q_{1}^{\mathrm{A}}$, is roughly by a factor of 40 . (We did not attempt to place the nodes at "good" points, as will be apparent in the sequel.) Moreover, interestingly, the troughs of one method seem to coincide with the peaks of the next. Fig. 1.1 is indicative of a large body of computational experience that we have assembled and which seems to imply that Filon-type methods are typically 
more accurate than asymptotic methods and that they can be made even more precise by an appropriate choice of extra quadrature nodes.

In Section 2 we explore practical means of monitoring the error in our methods for highly oscillatory quadrature. As is well known, the assessment of the error in Gaussian quadrature is fairly expensive, at least as much as the quadrature itself [2]. This is not the case with our methods, since they are based on asymptotic, rather than Taylor expansions. As turns out, their error can be monitored very precisely with very modest extra computational cost. This will go a long way towards explaining the behaviour observed in Fig. 1.1 and, in general, the superiority of Filon over the asymptotic method, as well as providing highly effective means toward error control.

The design of asymptotic and Filon-like methods of high asymptotic order requires the computation of derivatives of $f$ at the endpoints and stationary points. Although this can be often accomplished with great ease, there are obvious advantages in developing derivative-free methods. Motivated by the technique of Section 2, in Section 3 we introduce a new family of Filon-type methods that do not require the computation of derivatives, while allowing us to attain arbitrarily high asymptotic order.

\section{Error control of highly oscillatory quadrature}

We commence from the case $g^{\prime} \neq 0$, when the expansion (1.2) is valid. It follows at once from (1.3) that

$$
Q_{p}^{\mathrm{A}}[f]-I[f] \sim \frac{e_{p}^{\mathrm{A}}[f]}{\omega^{p+1}}+O\left(\omega^{-p-2}\right), \quad|\omega| \gg 1
$$

where

$$
e_{p}^{\mathrm{A}}[f]=\frac{\mathrm{e}^{\mathrm{i} \omega g(a)}}{g^{\prime}(a)} f_{p}(a)-\frac{\mathrm{e}^{\mathrm{i} \omega g(b)}}{g^{\prime}(b)} f_{p}(b)
$$

Therefore

$$
\Lambda_{-}^{\mathrm{A}}[f] \leq\left|e_{p}^{\mathrm{A}}[f]\right| \leq \Lambda_{+}^{\mathrm{A}}[f]
$$

where

$$
\Lambda_{-}^{\mathrm{A}}[f]=\left|\frac{\left|f_{p}(a)\right|}{\left|g^{\prime}(a)\right|}-\frac{\left|f_{p}(b)\right|}{\left|g^{\prime}(b)\right|}\right|, \quad \Lambda_{+}^{\mathrm{A}}[f]=\frac{\left|f_{p}(a)\right|}{\left|g^{\prime}(a)\right|}+\frac{\left|f_{p}(b)\right|}{\left|g^{\prime}(b)\right|} .
$$

In particular, in the important case of the Fourier oscillator $g(x)=x$ we have

$$
\Lambda_{-}^{\mathrm{A}}[f]=\left\|f^{(p)}(a)|-| f^{(p)}(b)\right\|, \quad \Lambda_{+}^{\mathrm{A}}[f]=\left|f^{(p)}(a)\right|+\left|f^{(p)}(b)\right| .
$$

Insofar as the Filon-type method (1.5) is concerned, we use our method of proof of the asymptotic order to argue that

$$
Q_{p}^{\mathrm{F}}[f]-I[f]=I[\tilde{f}-f] \sim \frac{e_{p}^{\mathrm{F}}}{\omega^{p+1}}+O\left(\omega^{-p-2}\right), \quad|\omega| \gg 1
$$

where

$$
e_{p}^{\mathrm{F}}[f]=\frac{\mathrm{e}^{\mathrm{i} \omega g(a)}}{g^{\prime}(a)}\left[\tilde{f}_{p}(a)-f_{p}(a)\right]-\frac{\mathrm{e}^{\mathrm{i} \omega g(b)}}{g^{\prime}(b)}\left[\tilde{f}_{p}(b)-f_{p}(b)\right]
$$


It is easy to verify from the definition of $f_{m}$, though, that

$$
f_{m}=\frac{f^{(m)}}{\left(g^{\prime}\right)^{m}}+\text { a linear combination of } f^{(k)} \text { for } k=0,1, \ldots, m-1 \text {. }
$$

Therefore, because of the Hermite interpolation conditions at the endpoints,

$$
e^{\mathrm{F}}[f]=\frac{\mathrm{e}^{\mathrm{i} \omega g(a)}}{\left[g^{\prime}(a)\right]^{p+1}}\left[\tilde{f}^{(p)}(a)-f^{(p)}(a)\right]-\frac{\mathrm{e}^{\mathrm{i} \omega g(b)}}{\left[g^{\prime}(b)\right]^{p+1}}\left[\tilde{f}^{(p)}(b)-f^{(p)}(b)\right] .
$$

We can again bracket the asymptotic error constant,

$$
\Lambda_{-}^{\mathrm{F}}[f] \leq\left|e_{p}^{\mathrm{A}}[f]\right| \leq \Lambda_{+}^{\mathrm{F}}[f]
$$

except that now

$$
\begin{aligned}
& \Lambda_{-}^{\mathrm{F}}[f]=\left|\frac{\left|\tilde{f}^{(p)}(a)-f^{(p)}(a)\right|}{\left|g^{\prime}(a)\right|^{p+1}}-\frac{\left|\tilde{f}^{(p)}(b)-f^{(p)}(b)\right|}{\left|g^{\prime}(b)\right|^{p+1}}\right|, \\
& \Lambda_{+}^{\mathrm{F}}[f]=\frac{\left|\tilde{f}^{(p)}(a)-f^{(p)}(a)\right|}{\left|g^{\prime}(a)\right|^{p+1}}+\frac{\left|\tilde{f}^{(p)}(b)-f^{(p)}(b)\right|}{\left|g^{\prime}(b)\right|^{p+1}} .
\end{aligned}
$$

We again single out the case $g(x)=x$, when

$$
\begin{aligned}
& \Lambda_{-}^{\mathrm{F}}[f]=|| \tilde{f}^{(p)}(a)-f^{(p)}(a)|-| \tilde{f}^{(p)}(b)-f^{(p)}(b) \|, \\
& \Lambda_{+}^{\mathrm{F}}[f]=\left|\tilde{f}^{(p)}(a)-f^{(p)}(a)\right|+\left|\tilde{f}^{(p)}(b)-f^{(p)}(b)\right| .
\end{aligned}
$$

To illustrate the power of these exceedingly simple error estimates, we refer again to Fig. 1.1. Now $f(x)=1 /(1+x), g(x)=x$ and $a=0, b=1$. It follows at once that

$$
\Lambda_{-}^{\mathrm{A}}[f]=\frac{3}{4}, \quad \Lambda_{+}^{\mathrm{A}}[f]=\frac{5}{4},
$$

faithfully confirming the behaviour of the top curve in Fig. 1.1. Moreover, we have the following error brackets for the four different Filon-type methods:

$$
\begin{aligned}
& \boldsymbol{c}=[0,1]: \quad \tilde{f}(x)=1-\frac{1}{2} x, \quad\left[\Lambda_{-}^{\mathrm{F}}, \Lambda_{+}^{\mathrm{F}}\right]=\left[\frac{1}{4}, \frac{3}{4}\right] ; \\
& c=\left[0, \frac{1}{2}, 1\right]: \quad \tilde{f}(x)=1-\frac{5}{6} x+\frac{1}{3} x^{2}, \quad\left[\Lambda_{-}^{\mathrm{F}}, \Lambda_{+}^{\mathrm{F}}\right]=\left[\frac{1}{12}, \frac{1}{4}\right] ; \\
& \boldsymbol{c}=\left[0, \frac{1}{3}, \frac{2}{3}, 1\right]: \quad \tilde{f}(x)=1-\frac{19}{20} x+\frac{27}{40} x^{2}-\frac{9}{40} x^{3}, \quad\left[\Lambda_{-}^{\mathrm{F}}, \Lambda_{+}^{\mathrm{F}}\right]=\left[\frac{1}{40}, \frac{3}{40}\right] ; \\
& \boldsymbol{c}=\left[0, \frac{1}{4}, \frac{1}{2}, \frac{3}{4}, 1\right]: \tilde{f}(x)=1-\frac{69}{70} x+\frac{13}{15} x^{2}-\frac{8}{15} x^{3}+\frac{16}{105} x^{4}, \quad\left[\Lambda_{-}^{\mathrm{F}}, \Lambda_{+}^{\mathrm{F}}\right]=\left[\frac{1}{140}, \frac{3}{140}\right] .
\end{aligned}
$$

Note thus that the troughs for the asymptotic method coincide with the peaks for the first Filon-type method and a similar phenomenon is valid for the first and the second Filon-type methods. This, however, is not true for the remaining methods and does not represent any general rule.

In general, while the error of an asymptotic method scales with the derivative of $f$ at the endpoints, for a Filon-type method $f$ need be replaced by the approximation error $\tilde{f}-f$. For most functions $f$ it is likely that the error of the interpolating polynomial is smaller than the function itself and that the interpolation error decreases once we are adding more points. This explains why so 


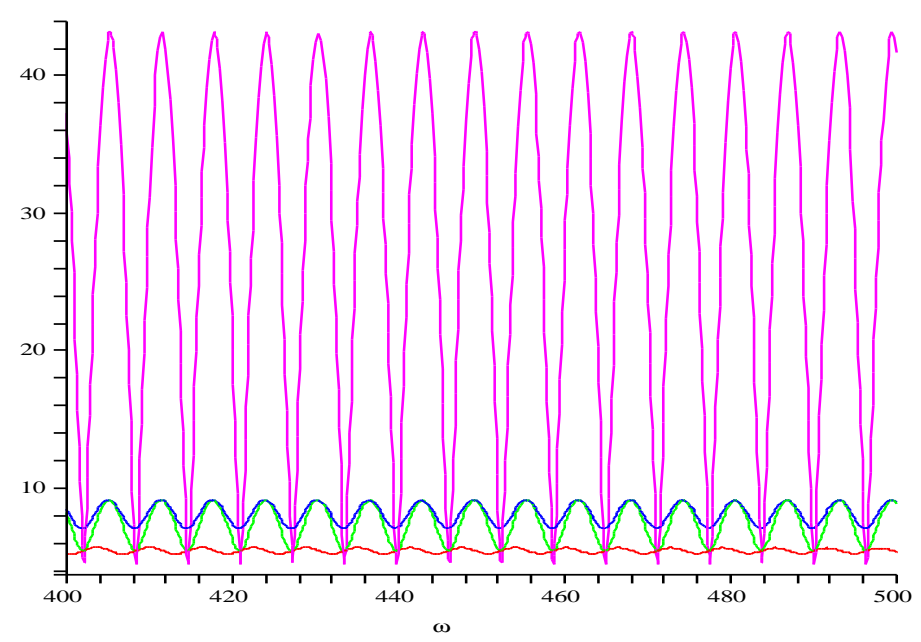

Figure 2.1: The error for $Q_{1}^{\mathrm{A}}$ (bottom) and three different Filon-type methods $Q_{1}^{\mathrm{F}}$ (from the second plot up), scaled by $\omega^{2}$, for $f(x)=\cos 10 x, g(x)=x$, $[a, b]=[0,1]$ and $400<\omega<500$.

often a Filon-type method is superior to an asymptotic method. However, this statement is far from universal. Thus, consider Fig. 2.1, where we have plotted the error for $f(x)=\cos 10 x, g(x)=x, a=0, b=1$. Now the pecking order is reversed: the asymptotic method wins and the quality of a Filon-type method deteriorates once we are adding more interpolation points!

The relative breakdown in a Filon-type method ("relative", since the method remains very precise) is closely related to the classical Runge phenomenon from interpolation theory [7], the failure of interpolation once derivatives increase too fast. In general, we can express the Hermite interpolation error for a smooth $f$ explicitly in the form

$$
\tilde{f}(x)-f(x)=\frac{1}{(m+1) !} f^{(m+1)}(\xi(x)) \prod_{l=1}^{\nu}\left(x-c_{l}\right)^{m_{l}+1},
$$

where $m=\sum_{l=1}^{\nu} m_{l}-1$ is the degree of $\tilde{f}$, while $\xi$ is a smooth function of $[a, b]$ to itself. Letting $m_{1}=m_{\nu}=p$ and mindful of

$$
\Lambda_{+}^{\mathrm{F}}[f]=\left|\tilde{f}^{(p)}(a)-f^{(p)}(a)\right|+\left|\tilde{f}^{(p)}(b)-f^{(p)}(b)\right|,
$$

we note that

$$
\tilde{f}^{(p)}(a)-f^{(p)}(a)=\frac{p !}{(m+1) !}(-1)^{p} f^{(m+1)}(\xi(a)) \prod_{l=2}^{\nu-1}\left(-c_{l}\right)^{m_{l}+1},
$$




\begin{tabular}{r|cccc}
\multicolumn{1}{c|}{$\omega$} & $Q_{1}^{\mathrm{A}}$ & 1 st $Q_{1}^{\mathrm{F}}$ & 2nd $Q_{1}^{\mathrm{F}}$ & 3rd $Q_{1}^{\mathrm{F}}$ \\
\hline 100 & $5.2717_{-4}$ & $5.2957_{-4}$ & $6.8647_{-4}$ & $8.7122_{-4}$ \\
1000 & $5.5252_{-6}$ & $6.5426_{-6}$ & $7.6610_{-6}$ & $2.0914_{-5}$ \\
10000 & $5.4372_{-8}$ & $9.0449_{-8}$ & $9.0717_{-8}$ & $4.2646_{-7}$
\end{tabular}

Table 2.1: The magnitude of the errors in the four methods of Fig. 2.1 for different values of $\omega$.
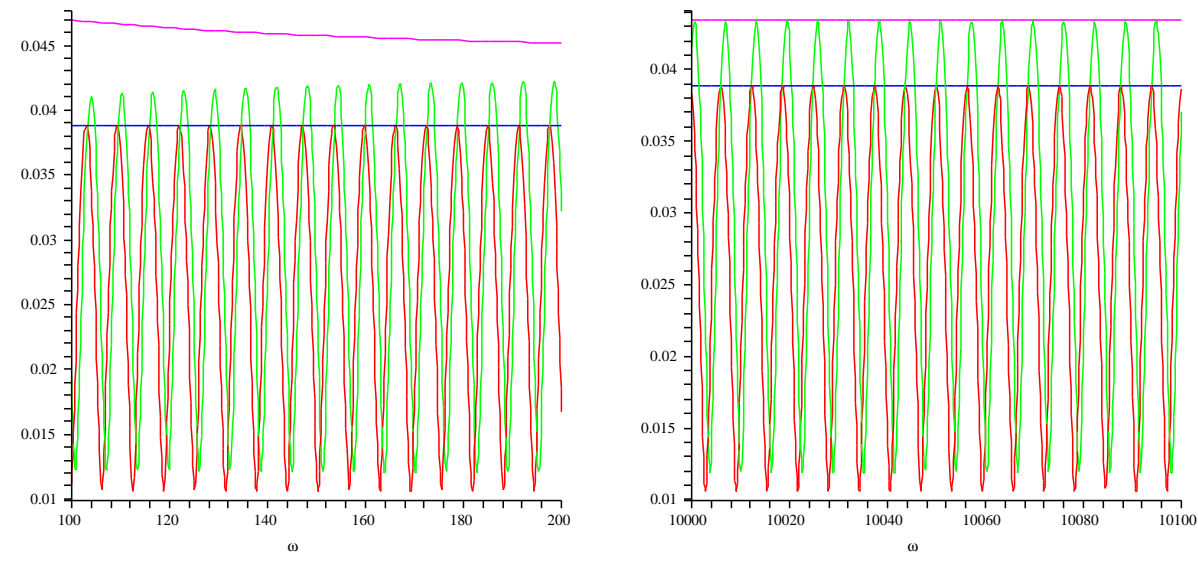

Figure 2.2: The error for $Q_{2}^{\mathrm{F}}[f], \boldsymbol{c}=\left[0, \frac{1}{4}, \frac{1}{2}, \frac{3}{4}, 1\right]$, scaled by $\omega^{3}$, and its estimate $\Lambda_{+}[f]$ (bottom oscillating curve and bottom line, respectively) and the same information for $Q_{2}^{\mathrm{aF}}[f]$. Here $f(x)=1 /\left(1+x^{2}\right), g(x)=x, a=0$ and $b=1$.

$$
\tilde{f}^{(p)}(b)-f^{(p)}(b)=\frac{p !}{(m+1) !} f^{(m+1)}(\xi(b)) \prod_{l=2}^{\nu-1}\left(1-c_{l}\right)^{m_{l}+1} .
$$

Therefore, a major determinant of the size of the error is the magnitude of the $(m+1)$ st derivative of $f$ near the endpoints. For functions $f$ such that $\left\|f^{(m+1)}\right\|_{\infty}$ grows rapidly with $m$ we can expect the asymptotic method to do better than Filon-type methods. However, such functions are an exception, rather than a rule, in applications and Filon-type methods are a good candidate, at least in the present setting, for methods of choice.

Of course, this discussion does not indicate that either of these methods is ineffective, in the presence of the Runge phenomenon, only that their pecking order is different. This is clear from Table 2.1.

To recap, we estimate the upper bound of $\left|Q_{p}[f]-I[f]\right|$, itself a rapidly oscillating function, by $\Lambda_{+}[f] / \omega^{p+1}$. Fig. 2.2 demonstrates how precise this estimate is, for two different ranges of $\omega$. The bottom oscillating curve there corresponds to the error of $Q_{2}^{\mathrm{F}}[f]$ with $\boldsymbol{c}=\left[0, \frac{1}{4}, \frac{1}{2}, \frac{3}{4}, 1\right], \boldsymbol{m}=[2,1,1,1,2]$, as applied to $I[f]=\int_{0}^{1} \mathrm{e}^{\mathrm{i} \omega x} /\left(1+x^{2}\right) \mathrm{d} x$, and scaled by $\omega^{3}$. The bottom horizontal line is 

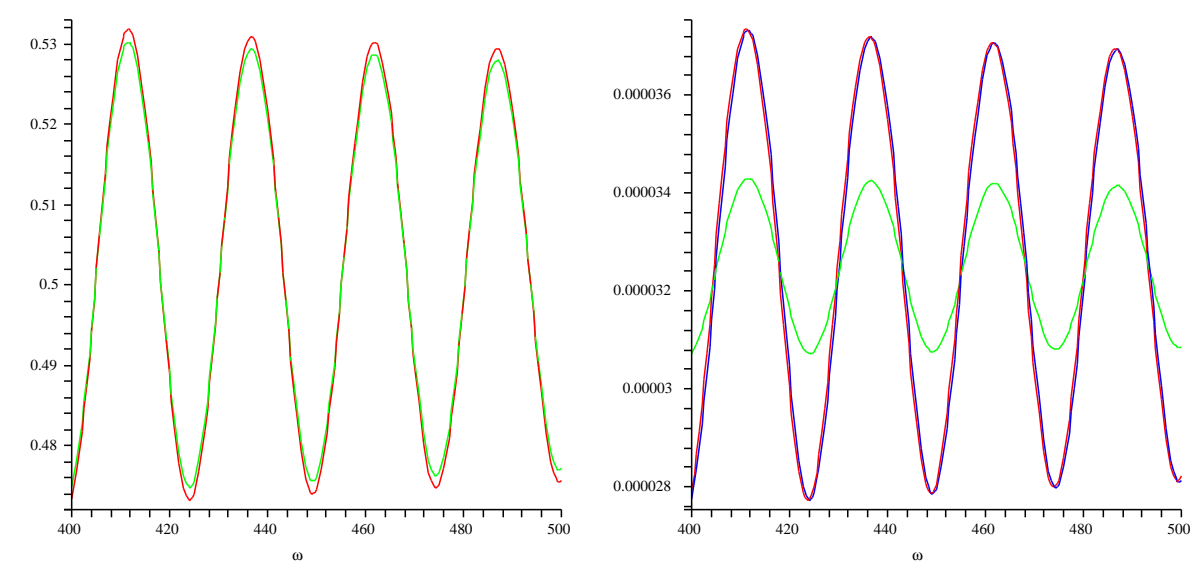

Figure 2.3: The error and its estimate for $Q_{2}^{\mathrm{A}}[f]$, both scaled by $\omega^{5 / 2}$ (on the left) and similar information (with two error estimates) for $Q_{2}^{\mathrm{F}}[f]$ with $\boldsymbol{c}=\left[0, \frac{1}{2}, 1\right], \boldsymbol{m}=[2,3,2]$. Here $f(x)=(1+x) \mathrm{e}^{x}, a=0, b=1$ and the oscillator $g(x)=x(1-x)$ has a critical point at $d=\frac{1}{2}$.

$\Lambda_{+}[f]$ and it is striking how well it estimates the peaks of the error. (We defer the introduction and discussion of the other method in this plot, $Q_{2}^{\mathrm{aF}}$, to the next section.) Similar behaviour has been observed in all computer experiments that we have attempted and the Runge phenomenon does not degrade the error estimate.

The principle of estimating the error does not change in the presence of stationary points. We assume again, for the sake of simplicity, a single stationary point $d \in(a, b)$, such that $g^{\prime}(d)=0, g^{\prime \prime}(d) \neq 0$, and employ the asymptotic expansion (1.7). It thus follows from (1.8) that

$$
Q_{p}^{\mathrm{A}}[f]-I[f] \sim \frac{\mu_{0}(\omega)}{(-\mathrm{i} \omega)^{p}} f_{p}(d)+O\left(\omega^{-p-1}\right),
$$

therefore we bound the error by

$$
\frac{\Lambda_{+}^{\mathrm{A}}[f]}{\omega^{p+\frac{1}{2}}}, \quad \text { where } \quad \Lambda_{+}^{\mathrm{A}}[f]=\omega^{1 / 2}\left|\mu_{0}(\omega) f_{p}(d)\right| .
$$

Note that $\left|\mu_{0}(\omega)\right|=O\left(\omega^{-1 / 2}\right)$ and that the "error constant" $\Lambda_{+}^{\mathrm{A}}[f]$ is not a constant anymore but a function of $\omega$. The lower error bound $\Lambda_{-}^{\mathrm{A}}[f]$ can be derived by similar means, but is of lesser practical importance.

Similarly, we let

$$
\Lambda_{+}^{\mathrm{F}}[f]=\omega^{1 / 2}\left|\mu_{0}(\omega)\left[\tilde{f}_{p}(d)-f_{p}(d)\right]\right|
$$

to estimate the error in Filon-type methods. Fig. 2.3 displays the errors and the error bounds, both scaled by $\omega^{5 / 2}$, for both $Q_{2}^{\mathrm{A}}$ and $Q_{2}^{\mathrm{F}}$. The error estimate for 


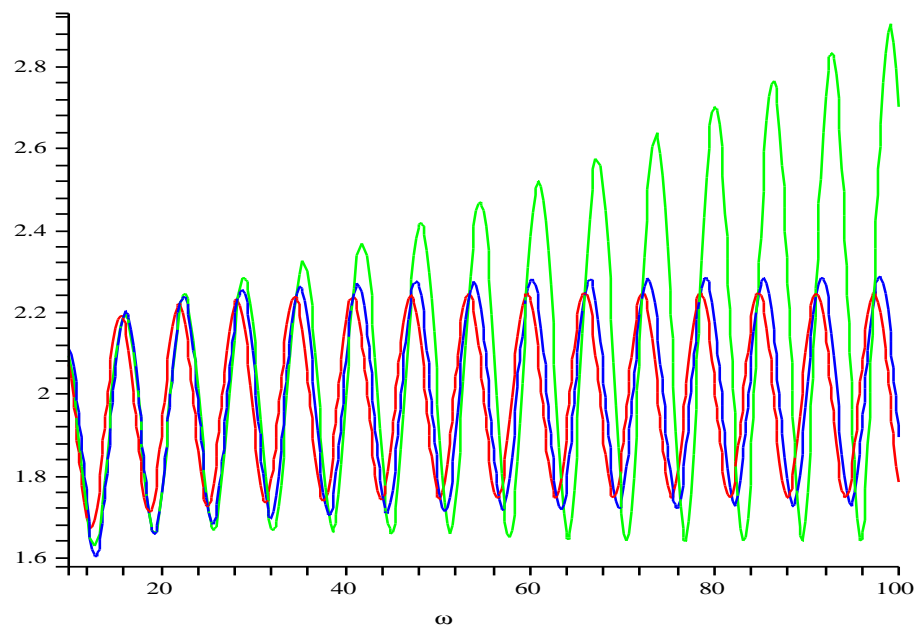

Figure 2.4: The "exact" error estimate in approximating $\int_{0}^{1} \mathrm{e}^{\mathrm{i} \omega x} /(1+x) \mathrm{d} x$ by $Q_{1}^{\mathrm{F}}, \boldsymbol{c}=[0,1]$, its estimate using an interpolating polynomial $\hat{f}$ (the increasing curve) and an adaptive polynomial $\hat{f}_{\omega}$ (virtually indistinguishable from the true error), all scaled by $\omega^{3}$.

the asymptotic method is virtually indistinguishable from the true error. The situation is somewhat different for the Filon-type method on the right. The error estimate is the "interior" curve, with the smaller amplitude. It is possible to show that the distance between the two curves attenuates like $O\left(\omega^{-1 / 2}\right)$, consistently with our theory, and for practical purposes this is an absolutely satisfactory error estimate. However, interestingly, a much superior error estimate can be obtained by adding the contribution of the $O\left(\omega^{-p-1}\right)$ term, originating on the boundary, i.e. letting

$\Lambda_{+}^{\mathrm{F}}[f]=\omega^{1 / 2}\left|\mu_{0}(\omega)\left[\tilde{f}_{p}(d)-f_{p}(d)\right]\right|+\frac{\omega^{-1 / 2}}{\left|g^{\prime}(a)\right|^{p}}\left|\tilde{f}_{p}(a)-f_{p}(a)\right|+\frac{\omega^{-1 / 2}}{\left|g^{\prime}(b)\right|^{p}}\left|\tilde{f}_{p}(b)-f_{p}(b)\right|$.

This results in an error estimate which in Fig. 2.3 is virtually identical to the true error.

Although tangential to our present argument, it is important to emphasize another observation from Fig. 2.3, namely the dramatic increase in the precision of the Filon-type method, as compared with the asymptotic method.

In this section we have demonstrated that a very effective error control of our methods for highly oscillatory quadrature is available at the cost of the evaluation of a derivative of the integrand at a small number of points. It is natural to ask, however, whether the computation of derivatives, which often is expensive, can be avoided altogether. An obvious idea is to form another interpolating polynomial, $\hat{f}$, say, of higher degree and, hopefully, better quality 
than $\tilde{f}$. In particular, we can add more interpolation nodes or increase the multiplicity of Hermite interpolation at existing points, thereby reusing function evaluations. This approach and its shortcoming is illustrated in Fig. 2.4. We compute there the integral

$$
I[f]=\int_{0}^{1} \frac{\mathrm{e}^{\mathrm{i} \omega x}}{1+x} \mathrm{~d} x
$$

thus neither stationary points nor the Runge phenomenon interfere with our calculations. We have estimated the error using derivatives at the endpoints and the (asymptotically) periodic curve stands for

$$
\omega^{3}\left|Q_{1}^{\mathrm{F}}[f]-I[f]-\frac{1}{\omega^{2}}\left\{\mathrm{e}^{\mathrm{i} \omega}\left[\tilde{f}^{\prime}(1)-f^{\prime}(1)\right]-\left[\tilde{f}^{\prime}(0)-f^{\prime}(0)\right]\right\}\right| .
$$

As the theory predicts, the error estimate recovers the $\omega^{-2}$ term exactly. Secondly, we have interpolated $f$ at four points by the cubic polynomial $\hat{f}$ : at the "old" points 0 and 1 , which have been already used to form the linear function $\tilde{f}$, as well as at $\frac{1}{20}$ and $\frac{19}{20}$ : the concentration of points near the boundary is motivated by the fact that, in absence of stationary points, it is the derivatives at the endpoints that make all the difference. The accuracy of this error estimate is reflected in the function

$$
\omega^{3}\left|Q_{1}^{\mathrm{F}}[f]-I[f]-\frac{1}{\omega^{2}}\left\{\mathrm{e}^{\mathrm{i} \omega}\left[\tilde{f}^{\prime}(1)-\hat{f}^{\prime}(1)\right]-\left[\tilde{f}^{\prime}(0)-\hat{f}^{\prime}(0)\right]\right\}\right|,
$$

which corresponds to the curve of growing amplitude in Fig. 2.4. For low(ish) values of $\omega$ we have a fairly good error estimate but it degrades as soon as $\omega^{-1}$ becomes large in comparison with the spacing $\frac{1}{20}$. This observation is very important and it is a key not just to a superior error estimate but to the derivativefree methods of the next section: allow the new interpolation points depend on $\omega$. Specifically, let us interpolate $f$ at the points $\left\{0, \frac{1}{\omega}, 1-\frac{1}{\omega}, 1\right\}$. Replacing $\hat{f}$ with the new interpolating cubic $\hat{f}_{\omega}$ produces a curve in Fig. 2.4 which is virtually indistinguishable from the error in the "exact" error estimate, using derivatives. The generalization of this "adaptive interpolation" to higher-order Filon methods and to stationary points is straightforward.

\section{Derivative-free quadrature methods}

Filon-type methods present an exceedingly efficient means of the computation of a highly oscillatory integral (1.1). However, once we wish to obtain asymptotic order $p>1$ ( or $p>1 / s$ in the presence of stationary points of degree $s \geq 1$, i.e. such that $\left.g^{\prime}(d)=\cdots=g^{(s-1)}(d)=0, g^{(s)}(d) \neq 0\right)$ we must compute derivatives at the endpoints and stationary points. Moreover, the number of derivatives required at a stationary point scales with its degree: to attain asymptotic order $p-1+\frac{1}{s}, p \geq 1$ we need to compute just $p-1$ derivatives at the endpoints but $(p-1) s$ derivatives at $d$. Sometimes this does not present a significant problem since either derivatives are available or can be computed exactly, e.g. with an automatic differentiation package. This, however, does not extend to the totality of all problems of interest. Often derivatives are not available (for example, 
when $f$ itself originates in a different numerical calculation) or are expensive or awkward to compute. Fortunately, it is possible to attain arbitrarily high asymptotic order without any derivatives whatsoever.

The first, somewhat naive, idea is to approximate derivatives by finite differences. This is equivalent to the placement of interpolation points very near to endpoints and stationary points. Indeed, it follows from the discussion in Section 2 that a good upper bound for $\Lambda_{+}^{\mathrm{F}}[f]$ in the absence of stationary points is

$$
\frac{p !}{(m+1) !}\left\|f^{(m+1)}\right\|_{\infty}\left[\prod_{l=2}^{\nu-1}\left(c_{l}-a\right)^{m_{l}+1}+\prod_{l=2}^{\nu-1}\left(b-c_{l}\right)^{m_{l}+1}\right] .
$$

Thus, if $c_{2}$, say, is near to $a$ and $c_{\nu-1}$ to $b$, we can render the leading error term very small. (Of course, the "best" course of action is to let $c_{2}=a, c_{\nu-1}=b$, but all this means is that we compute - exactly - extra derivatives at the endpoints, something that we are trying to avoid.)

An alternative is to form finite-difference approximations to derivatives at points that themselves depend upon $\omega$. Thus, instead of interpolating to $f^{(k)}\left(c_{l}\right)$, $k=0,1, \ldots, m_{l}-1$, say, we interpolate just to $f$ at the points $c_{l}-\gamma_{l, j} / \omega$, $j=0,1, \ldots, m_{l}-1$. The simplest course of action, which we pursue in this paper, is to choose a sufficiently small $\gamma>0$ ("smallness" depends on the size of $\omega$, since we need to fit all the nodes in $[a, b]$ : for large $\omega$ letting $\gamma=1$ will do) and use the following interpolation points:

$$
\begin{aligned}
c_{1}=a: & a+\frac{\gamma}{\omega} k, & k & =0,1, \ldots, m_{1}-1, \\
c_{l} \in(a, b): & c_{l}+\frac{\gamma}{\omega} k, & k & =-\left\lfloor\frac{m_{l}-1}{2}\right\rfloor, \ldots,\left\lfloor\frac{m_{l}}{2}\right\rfloor, \\
c_{\nu}=b: & b-\frac{\gamma}{\omega} k, & k & =0,1, \ldots, m_{\nu}-1 .
\end{aligned}
$$

The values of $f$ therein are interpolated by the $m$ th degree polynomial $\tilde{f}_{\omega}$, where, as before, $m=\sum_{l=1}^{\nu} m_{l}-1$, and we set

$$
Q_{p}^{\mathrm{aF}}[f]=\int_{0}^{1} \tilde{f}_{\omega}(x) \mathrm{e}^{\mathrm{i} \omega g(x)} \mathrm{d} x,
$$

where $p=\min \left\{m_{1}, m_{\nu}\right\}$. We call (3.1) an adaptive Filon-type method.

It is elementary to observe that

$$
\tilde{f}_{\omega}^{(k)}\left(c_{l}\right)=f^{(k)}\left(c_{l}\right)+O\left(\omega^{-m_{l}+k}\right)
$$

for all $k=0,1, \ldots, m_{l}$ and $l=1,2, \ldots, \nu$. Thus, suppose again that the interpolation points in the vicinity of $c_{l}$ are $c_{l}+\gamma_{l, j} / \omega, j=0,1, \ldots, m_{l}-1$, where the $\gamma_{l, j}$ s are distinct. Then

$$
\tilde{f}_{\omega}(x)-f(x)=v_{l}(x) \prod_{j=0}^{m_{l}-1}\left(x-c_{l}+\frac{\gamma_{l, j}}{\omega}\right),
$$

where

$$
v_{l}(x)=\frac{1}{(m+1) !} f^{(m+1)}(\eta(x)) \prod_{\substack{r=1 \\ r \neq l}}^{\nu} \prod_{j=0}^{m_{r}-1}\left(x-c_{r}+\frac{\gamma_{r, k}}{\omega}\right) .
$$


We first note that

$$
\tilde{f}_{\omega}\left(c_{l}\right)-f\left(c_{l}\right)=\omega^{-m_{l}} v_{l}\left(c_{l}\right) \prod_{j=0}^{m_{l}-1} \gamma_{l, j}=O\left(\omega^{-m_{l}}\right) .
$$

Next,

$$
\begin{aligned}
\tilde{f}_{\omega}^{\prime}(x)-f^{\prime}(x) & =v_{l}(x) \sum_{s=0}^{m_{l}-1} \prod_{j \neq s}\left(x-c_{j}+\frac{\gamma_{l, j}}{\omega}\right)+v_{l}^{\prime}(x) \prod_{j=0}^{m_{l}-1}\left(x-c_{l}+\frac{\gamma_{l, j}}{\omega}\right) \\
\Rightarrow \quad \tilde{f}_{\omega}^{\prime}\left(c_{l}\right)-f^{\prime}\left(c_{l}\right) & =\omega^{-m_{l}+1} v_{l}\left(c_{l}\right) \sum_{s=0}^{m_{l}-1} \prod_{j \neq s} \gamma_{l, j}+O\left(\omega^{-m_{l}}\right)
\end{aligned}
$$

and, by the same token,

$$
\begin{aligned}
\tilde{f}_{\omega}^{\prime \prime}\left(c_{l}\right)-f^{\prime \prime}\left(c_{l}\right) & =\omega^{-m_{l}+2} v_{l}\left(c_{l}\right) \sum_{s_{1}=0}^{m_{l}-1} \sum_{\substack{s_{2}=0 \\
s_{2} \neq s_{1}}}^{m_{l}-1} \prod_{j \neq s_{1}, s_{2}} \gamma_{l, j}+O\left(\omega^{-m_{l}+1}\right), \\
\tilde{f}_{\omega}^{\prime \prime \prime}\left(c_{l}\right)-f^{\prime \prime \prime}\left(c_{l}\right) & =\omega^{-m_{l}+3} v_{l}\left(c_{l}\right) \sum_{s_{1}=0}^{m_{l}-1} \sum_{\substack{s_{2}=0 \\
s_{2} \neq s_{1}}}^{m_{l}-1} \sum_{\substack{s_{3}=0 \\
s_{3} \neq s_{1}, s_{2}}}^{m_{l}-1} \prod_{j \neq s_{1}, s_{2}, s_{3}} \gamma_{l, j}+O\left(\omega^{-m_{l}+2}\right)
\end{aligned}
$$

and so on: the general proof is clear, although exceedingly tedious.

Since each $f_{n}$ is a linear combination of $f, f^{\prime}, \ldots, f^{(n)}$, we thus deduce that

$$
\tilde{f}_{\omega_{n}}\left(c_{l}\right)-f_{n}\left(c_{l}\right)=O\left(\omega^{-m_{l}+n}\right), \quad n=0,1, \ldots, m_{l} .
$$

Consequently, for every $n$ in this range

$$
\frac{1}{(-\mathrm{i} \omega)^{n}}\left[\tilde{f}_{\omega_{n}}\left(c_{l}\right)-f\left(c_{l}\right)\right]=O\left(\omega^{-m_{l}}\right)
$$

It now follows from (1.2) and (1.7) that the adaptive Filon-type method (3.1) has always the same asymptotic order as the corresponding Filon-type method. Hence, we can dispense altogether with the computation of derivatives.

A striking comparison between a non-adaptive approximation of derivative by finite differences and an adaptive Filon-type method is provided by Fig. 3.1. We have used there two derivative-free methods to approximate

$$
\int_{0}^{1} \frac{2-x}{2+x} \mathrm{e}^{\mathrm{i} \omega x} \mathrm{~d} x=\frac{1-\mathrm{e}^{\mathrm{i} \omega}}{\omega}+4 \mathrm{e}^{-2 \mathrm{i} \omega}[\operatorname{Ei}(1,-2 \mathrm{i} \omega)-\operatorname{Ei}(1,-3 \mathrm{i} \omega)],
$$

where $\mathrm{Ei}$ is the exponential integral [1]. The first is a Filon-type method with the interpolation points $\boldsymbol{c}=\left[0, \frac{1}{1000}, \frac{999}{1000}, 1\right]$. This might appear to be a fine enough spacing to approximate well first derivatives at the endpoints and, indeed, as the figure on the left demonstrates, for moderate values of $\omega$ the error behaves like $O\left(\omega^{-3}\right)$. Yet, for larger $\omega$, as can be seen in the figure on the right, the 

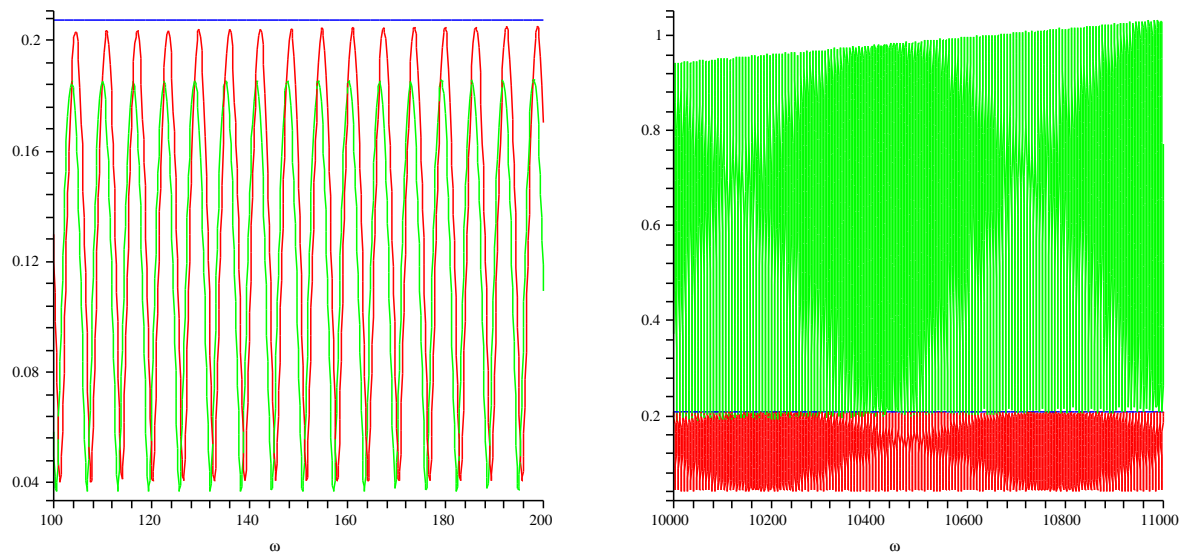

Figure 3.1: The error, scaled by $\omega^{3}$, committed by two methods: Filon-type with the nodes $\left[0, \frac{1}{1000}, \frac{999}{1000}\right]$ and multiplicities $\boldsymbol{m}=[1,1,1,1]$ (the fainter line style) and adaptive Filon-type with the nodes $[0,1]$, multiplicities $[2,2]$ and $\gamma=1$ (the bolder line), as well as the error bound for the latter (the almost-horizontal line).

error is just $O\left(\omega^{-2}\right)$, consistently with asymptotic order 1 . The second method in Fig. 3.1 is the adaptive Filon-type method with $\boldsymbol{c}=[0,1]$, multiplicities $[2,2]$ and $\gamma=1$ : in other words, we interpolate $f$ at $0, \frac{1}{\omega}, 1-\frac{1}{\omega}$ and 1 . The outcome, represented by the bolder line style, is an error which, regardless of the size of $\omega$, is of an asymptotic order 2 . We note in passing that for small $\omega>0$ the error of the first method is actually smaller than that of the adaptive method. This makes a perfect sense. The error in both methods consists of two components: firstly, how well are derivatives at the endpoints approximated by finite differences and, secondly, how large is the quadrature error with exact derivatives (i.e., the error of the Filon-type method with $c=[1,1]$ and multiplicities $\boldsymbol{m}=[2,2])$. For small $\omega>0$ the finite-difference approximation of derivatives by the first method is clearly superior, but it deteriorates fast when $\omega^{-1}$ is no longer large in comparison with $\frac{1}{1000}$. On the other hand, the adaptive method adjusts the finite-difference approximation to the size of $\omega$, rendering it uniformly small regardless of the frequency.

Estimating the error of an adaptive Filon-type method, we clearly wish to avoid the calculation of derivatives of $f$. This prevents us from using the main approach of Section 2, namely to form error bounds using derivatives of the interpolation error at the endpoints and at critical points. Instead, we adopt the alternative approach of replacing $f$ with an interpolating polynomial $\hat{f}_{\omega}$. We form $\hat{f}_{\omega}$ by adding extra interpolation points, as necessary, about endpoints and stationary points. Thus, referring back to Fig. 3.1, we take $\boldsymbol{c}=[0,1]$ and $\boldsymbol{m}=[3,3]$. In other words, we interpolate $f$ at $0, \frac{1}{\omega}, \frac{2}{\omega}, 1-\frac{2}{\omega}, 1-\frac{1}{\omega}, 1$ by a quintic. 
For a method of an asymptotic order $p$ we estimate

$$
\max _{\omega \gg 1}\left|Q_{p}^{\mathrm{aF}}[f]-I[f]\right| \approx \frac{\Lambda_{+}^{\mathrm{aF}}[f]}{\omega^{p}},
$$

with obvious adjustments once, in the presence of stationary points, the asymptotic order is noninteger. Forming $\Lambda_{+}^{\mathrm{aF}}$, which itself depends weakly upon $\omega$, we must take into account both the quadrature error, as given by the asymptotic expansions, and interpolation error in replacing derivatives of $f$ by finite differences, but we disregard the error in replacing $f$ with $\hat{f}_{\omega}$ in an asymptotic expansion. Reverting to the method from Fig. 3.1, we thus have

$$
\begin{aligned}
Q_{2}^{\mathrm{aF}}[f]-I\left[\hat{f}_{\omega}\right]= & I\left[\tilde{f}_{\omega}-\hat{f}_{\omega}\right] \\
= & -\frac{1}{(-\mathrm{i} \omega)^{2}}\left\{\mathrm{e}^{\mathrm{i} \omega}\left[\tilde{f}_{\omega}^{\prime}(1)-\hat{f}_{\omega}^{\prime}(1)\right]-\left[\tilde{f}_{\omega}^{\prime}(0)-\hat{f}_{\omega}^{\prime}(0)\right]\right\} \\
& -\frac{1}{(-\mathrm{i} \omega)^{3}}\left\{\mathrm{e}^{\mathrm{i} \omega}\left[\tilde{f}_{\omega}^{\prime \prime}(1)-\hat{f}_{\omega}^{\prime \prime}(1)\right]-\left[\tilde{f}_{\omega}^{\prime \prime}(0)-\hat{f}_{\omega}^{\prime \prime}(0)\right]\right\}+O\left(\omega^{-4}\right),
\end{aligned}
$$

motivating the choice

$$
\begin{aligned}
\Lambda_{+}^{\mathrm{aF}}[f]= & \left|\left[\tilde{f}_{\omega}^{\prime \prime}(1)-\hat{f}_{\omega}^{\prime \prime}(1)\right]-\mathrm{i} \omega\left[\tilde{f}_{\omega}^{\prime}(1)-\hat{f}_{\omega}^{\prime}(1)\right]\right| \\
& +\left|\left[\tilde{f}_{\omega}^{\prime \prime}(0)-\hat{f}_{\omega}^{\prime \prime}(0)\right]-\mathrm{i} \omega\left[\tilde{f}_{\omega}^{\prime}(0)-\hat{f}_{\omega}^{\prime}(0)\right]\right|
\end{aligned}
$$

The curve $\Lambda_{+}^{\mathrm{fA}}[f]$ is depicted in Fig. 3.1: this is the virtually-horizontal line which on the left lies a whisker above the scaled error of the adaptive method and on the right is almost exactly on top of the error curve. The tightness of the approximation is evident.

Another example of an adaptive Filon-type method is presented in Fig. 2.2. There we compare a "classical" Filon-type method with $\boldsymbol{c}=\left[0, \frac{1}{4}, \frac{1}{2}, \frac{3}{4}, 1\right], \boldsymbol{m}=$ $[2,1,1,1,2]$ and the adaptive Filon-type method with the same parameters. The error of the adaptive method is about $13 \%$ larger: this is the price we are paying for replacing derivatives with adaptive finite differences. Yet, the feature to observe is the error bound $\Lambda_{+}^{\mathrm{aF}}[f]$, the upper nonoscillatory curve. For lower frequencies, on the left, it somewhat overshoots the true error bound. Moreover, the curve is not horizontal, a tell-tale sign that it is not yet in its asymptotic stage. However, on the right, in a high-frequency regime, the upper error bound is estimated virtually perfectly.

\section{Conclusions}

Practical implementation of quadrature methods for highly oscillatory integrals requires good upper bounds on the error. They are instrumental in ensuring that the error falls below a user-provided tolerance and that this desirable state of affairs is attained with the least investment of computational resources.

In this paper we have presented an error-control technique for two methods that have been introduced in [5] and which probably represent the state of the art in the integration of highly oscillatory integrals (1.1).

Motivated by our error-control mechanism, we have introduced a variant of the Filon-type technique which, by allowing quadrature points to depend on 
the frequency $\omega$, produces the same asymptotic order (and roughly the same quality of computation) without using derivatives altogether. The error in the new methods can be bounded effectively, again without using derivatives.

\section{REFERENCES}

1. M. Abramowitz And I. A. Stegun, eds., Handbook of Mathematical Functions, National Bureau of Standards, Washington, DC, 1964.

2. P. J. Davis And P. Rabinowitz, Methods of Numerical Integration, Academic Press, Orlando, FL, 2nd ed., 1980.

3. L. N. G. Filon, On a quadrature formula for trigonometric integrals, Proc. Royal Soc. Edinburgh, 49 (1928), pp. 38-47.

4. A. ISERLES, On the numerical quadrature of highly-oscillating integrals II: Irregular oscillators, Tech. Rep. NA2003/09, DAMTP, University of Cambridge, 2003.

5. A. IsERLES AND S. P. NøRSETt, Efficient quadrature of highly oscillatory integrals using derivatives, Tech. Rep. NA2004/03, DAMTP, University of Cambridge, 2004.

6. F. W. J. Olver, Asymptotics and Special Functions, Academic Press, New York, 1974.

7. M. J. D. Powell, Approximation Theory and Methods, Cambridge University Press, Cambridge, 1981.

8. E. Stein, Harmonic Analysis: Real-Variable Methods, Orthogonality, and Oscillatory Integrals, Princeton University Press, Princeton, NJ, 1993. 